\title{
PREPROCESSING OF HOPKINSON BAR EXPERIMENT DATA: FILTER ANALYSIS
}

\author{
Marcel Adorna*, Jan Falta, Tomáš Fíla, Petr Zlámal \\ Czech Technical University in Prague, Faculty of Transportation Sciences, Department of Mechanics and \\ Materials, Konviktská 20, 12000 Prague 1, Czech Republic \\ * corresponding author: adorna@fd.cvut.cz
}

\begin{abstract}
This work presents a data preprocessing procedure for signal acquired during high strain-rate loading using a custom Split Hopkinson Pressure Bar (SHPB). Before the evaluation of the experimental data, preprocessing of the measured signals including application of suitable digital or analog filter needs to be performed. Our department mainly focuses on measurements performed on advanced materials (e.g. materials with predefined structures or hybrid foams). For such measurements, it is essential to perform data preprocessing and apply suitable filter, to be able to appropriately determine deformation pulses on the measuring bars. This paper focuses foremost on spectral analysis of the measured signals, and design of optimal method of data filtering. Data from several different SHPB experiments were processed and results of different filtering methods are shown in this paper. Parameters of the best performing filter were optimized and shown to be universal for wide range of SHPB measurements.
\end{abstract}

KEYWORDS: SHPB, filter analysis, strain-gage measurements, Chebyshev filter.

\section{INTRODUCTION}

Recent industrial development shows an increase in industrial applications of advanced materials and structures. For example, in transportation industry complex materials providing high absorption of impact energy together with low density are required. Materials with predefined structures produced using various additive manufacturing methods (e. g. materials with auxetic behaviour) or hybrid foams (e. g. polymeric core with metal coating) show great potential in various energy absorption applications [1]. By using these types of materials, it is possible to significantly improve efficiency and safety of constructions or vehicles. However, proper description of their complex structural and mechanical properties might be very challenging due to their complicated structures, especially in case of high strain-rate loading [2]. Therefore, it is essential to not only perform precise high velocity material tests but also develop advanced methods that would enable reliable evaluation of such experimental data. Split Hopkinson pressure bar (SHPB) technique is well established method for investigation of deformation behaviour of conventional materials (e. g. bulk metallic materials) under high strain-rate loading. For experiments with soft materials and materials with complex structures, a modified SHPB (usually with in-house design and application tailored properties) together with custom evaluation software is required 3 . For cellular materials, it is shown that precise application of suitable filter is essential, and relevance of the evaluated results depends on proper pre-processing of the experimental data. Experimental setup might differ in terms of used measurement bars (usually alluminum or PMMA bars are used), length of used striker (up to $500 \mathrm{~mm}$ ), impact velocity of used striker (up to $50 \mathrm{~ms}^{-1}$ ), number and type of strain gauges (both foil and semiconductor strain gauges are used) and their position on measuring bars. Pre-processing procedure may therefore differ according to the used experimental setup and different type of filter (e.g. Butterworth filter, Chebyshev filter etc.) might suit specific experimental data.

Measured signals from SHPB experiments are subject to high frequency noise and contain various peak values (e. g. valve release interference peak). Due to this, it is important to apply a suitable filter to process the data before its further evaluation. However, proper filter analysis and spectral analysis of measured signals is needed. In this paper, signals from several SHPB measurements using different stiker lengths and impact velocities of strikers were analysed. Optimal filter was found, one which best suits very specific requirements of SHPB data, such as filtering only identified noise frequencies while not affecting properties of measured elastic waves which would lead to unprecise experimental results.

\section{MATERIALS AND METHODS}

\subsection{Split Hopkinson Pressure Bar}

Basic scheme of Split Hopkinson Presure Bar (SHPB) is shown in Fig. 1. All experiments were performed using a custom Kolsky SHPB setup (overview shown in Fig. 2). Both bars and the striker have the same diameter $20 \mathrm{~mm}$. High-strength aluminium alloy (ENAW 7075) was used as a material of the bars. The striker was accelerated using a gas-gun system with 16 bar maximum pressure. The incident and the trans- 


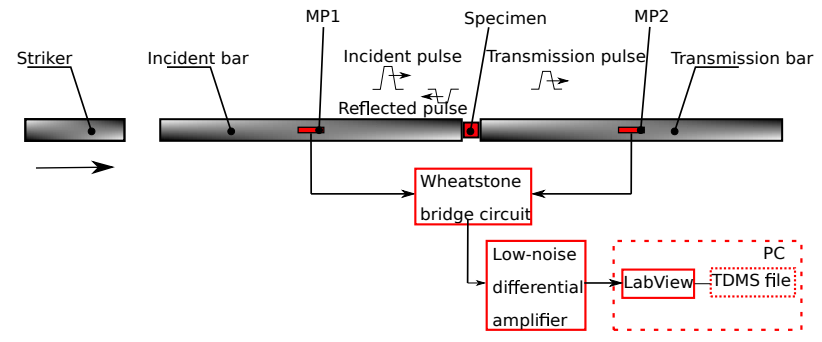

Figure 1. Basic scheme of SHPB

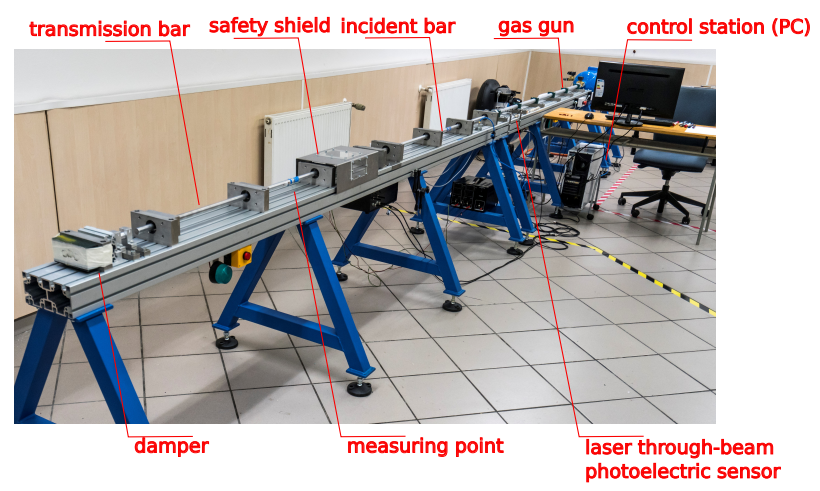

FiguRE 2. Loading device overview

mission bar had the same length $1600 \mathrm{~mm}$ and were guided and supported by set of a low-friction polymerliner slide bearings (Drylin FJUM, IGUS, Germany) with a custom stainless steel housing. All surfaces of both bars and the striker were carefully grounded and polished to ensure a smooth motion of the bars in the bearings and achieve plan-parallerism of the bars faces.

\subsection{Strain Gauge measurement}

Elastic strain waves were measured on two measuring points equipped with foil strain gauges in Wheatstone half-bridge arrangement during the test. Active differential low noise amplifier (EL-LNA-2, Elsys AG, Switzerland) with gain 100 was used to amplify strain gauge signal before the digitization. The strain gauge signal was sampled using 16-bit digitizer (PCI-9826H, ADLINK Technology, Inc., Taiwan) with maximal $20 \mathrm{MHz}$ sample rate to maximize signal-to-noise ratio of measured signals. However, all signals were subject to the high frequency noise as well as white Gaussian noise of different amlitudes and contained various peak values (e. g. valve release interference peak) which were not significant for the experiment evaluation.

\subsection{Auxetic structures}

Filter analysis and optimisation was performed on the measured signals from experiments performed with a group of so called auxetic materials with predefined periodic structure. These materials exhibit in-plane negative strain-dependent Poisson's ratio. Selective laser sintering (SLS) method was used for production of the samples and manufacturing was performed using
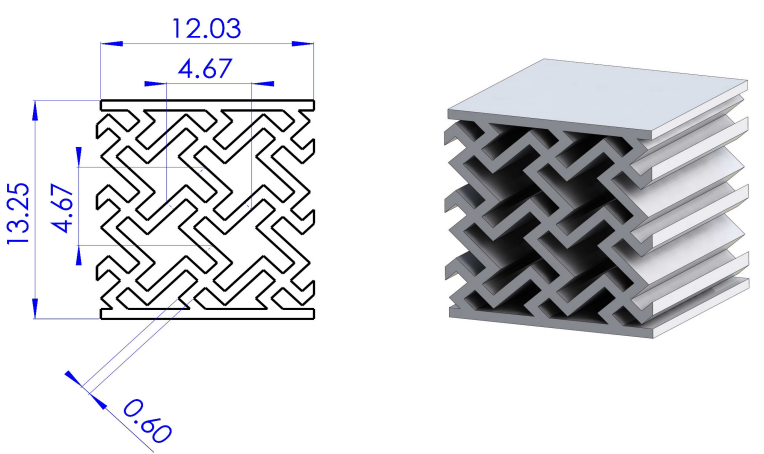

FiguRE 3. 2D missing-rib auxetic structure

the AM250 system (Renishaw, UK) by sintering the 316L-0407 powdered austenitic steel [4]. Structure with missing-rib unit-cell geometry (i.e. 2D missingrib) is shown in Fig. 3 Resulting optimized filter is however to be universal for all measurements of cellular materials (with similar material properties as auxetics) preformed using our aluminium SHPB bar setup.

\section{MeAsured SignAl ANALYSIS}

Analysis of the measured signals was performed using Matlab environment (Mathworks Inc.). Spectral analysis of signals was carried out using Fast Fourier Transform. Three different types of filters implemented in Matab were tested and evaluation of filter performance was carried out to select the best filter for the chosen experimental setup. An exemplary elastic wave signal with applied filter is shown in Fig. 4

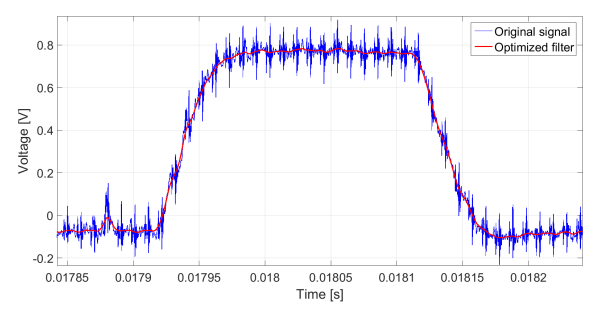

FiguRE 4. Elastic wave signal with applied filter

Periodograms of measured signals were obtained using Matlab tool to investigate main noise frequencies present in the signal. Note that due to used sampling frequency of $20 \mathrm{MHz}$, axes of periodograms obtained using Matlab function do not directly correspond with noise frequencies, but can be easily derived from it. Influence of boundary conditions of the experiment (impact velocity, striker length etc.) on measured signals and its noise are investigated.

\subsection{MEASURING BARS SIGNALS}

Periodograms of measured signals on incident and transmission bar are shown in Fig. 5. It is shown, that for both incident and transmission signal, the main 
noise frequencies were the same, which suggests that noise properties were also the same and there were no additional vibrations caused by the bars contact. It is also shown, that the main noise frequencies were of singular character and could be attenuated using a specific notch filter. In the range from $2 \mathrm{MHz}$ to $4 \mathrm{MHz}$ all frequencies also showed noisy character, which could be attenuated using band-stop filter.

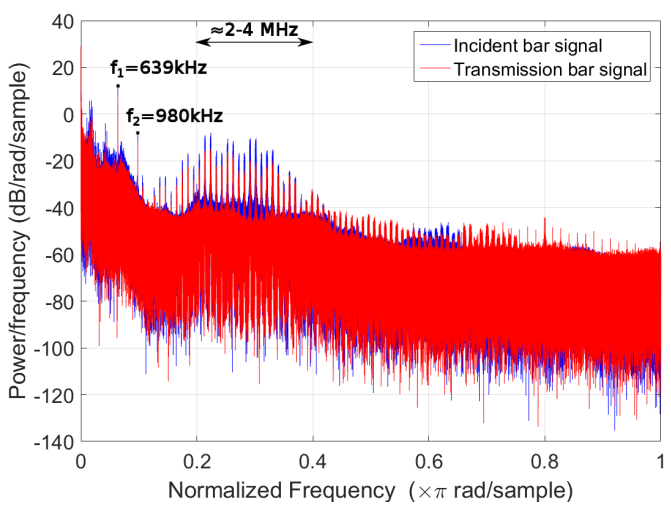

Figure 5. Periodogram of incident and transmission signal: $l_{s}=500 \mathrm{~mm}$ (long striker), $v_{s}=20.85 \mathrm{~ms}^{-1}$ (low striker velocity)

\subsection{STRIKER LENGTH}

Periodograms of representative measured signals from experiments performed using short and long striker are shown in Fig. 6. Main noise frequencies were again almost the same and it was shown, that signal noise is independent on length of used striker during SHPB experiment.

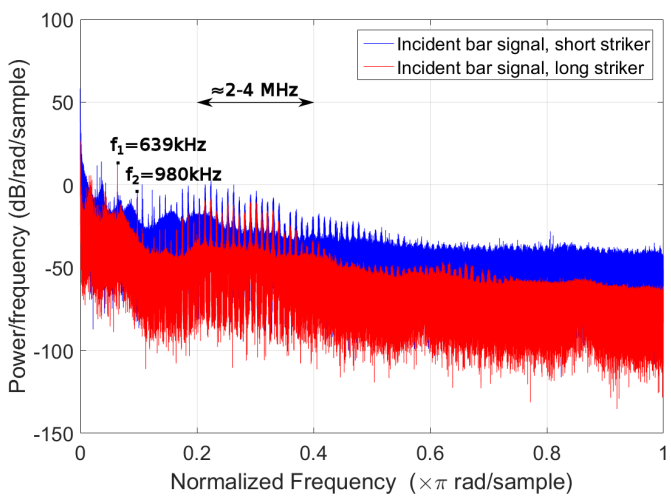

Figure 6. Periodogram of incident signal: $l_{s}=$ $500 \mathrm{~mm}$ (long striker), $v_{s}=24.05 \mathrm{~ms}^{-1}$ (low striker velocity) and $l_{s}=100 \mathrm{~mm}$ (short striker), $v_{s}=$ $19.32 \mathrm{~ms}^{-1}$ (low striker velocity)

\subsection{STRIKER VELOCITY}

Experiments with wide range of striker velocities were performed to determine whether measured signals and its noise depends on striker impact velocity. Periodogram of measured signals from experiments performed using high and low striker velocity are shown on Fig 7 It is shown, that main noise frequencies are again almost identical and therefore it can be assumed that properties of measured noise are independent on striker velocity.

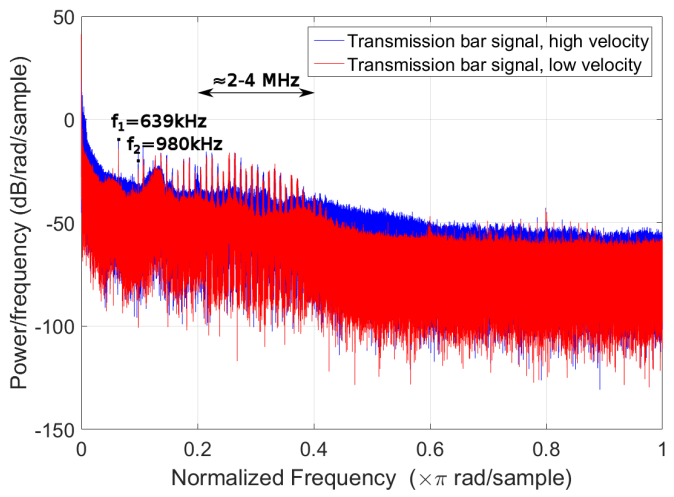

Figure 7. Periodogram of incident signal: $l_{s}=$ $500 \mathrm{~mm}$ (long striker), $v_{s}=45.21 \mathrm{~ms}^{-1}$ (high striker velocity) and $l_{s}=500 \mathrm{~mm}$ (long striker), $v_{s}=$ $21.03 \mathrm{~ms}^{-1}$ (low striker velocity)

\section{Filter ANALYSis}

Filter analysis and evaluation of filter performance was carried out to select the best and universal filter for the used SHPB setup. Main goal of data pre-processing was to get rid of high frequency noise while preserving measured signals undeformed and thus minimize of the negative effect of filtration on experimental results. Both low-pass Butterworth analog filter and digital Savitzky-Golay filter were being used for SHPB signals filtering. These two filters and their performance were compared with designed optimal filter based on inverse Chebyshev procedure implemented in Matlab.

\subsection{Butterworth FiLter}

Low-pass Butterwoth filter was used in previous studies performed on our department due to its maximally flat frequency response in both passband and stopband. Hovewer, in order to filter signals in a satisfying manner, stopband of Butterworth filter and its corresponding attenuation deformed measured signal too much and important parts of the experimental data were often lost.

\subsection{SAVitZKY-Golay Digital FilteR}

The Savitzky-Golay filters are usually used for data smoothing and denoising in the spectral analysis, signal processing, image processing field etc. Its basic principle is based on the least square error fitting between original data and polynomial function with predefined degree inside a moving window [5, 6, Initial setting of these two parameters thus have an immense influence on smoothing procedure and its outcome. This also means that these predefined parameters correspond with signal-to-noise ratio of measured signals (See also [7]) and therefore it is necessary to redefine these parameters separately for each measurement in order to smooth the data in similar way. 

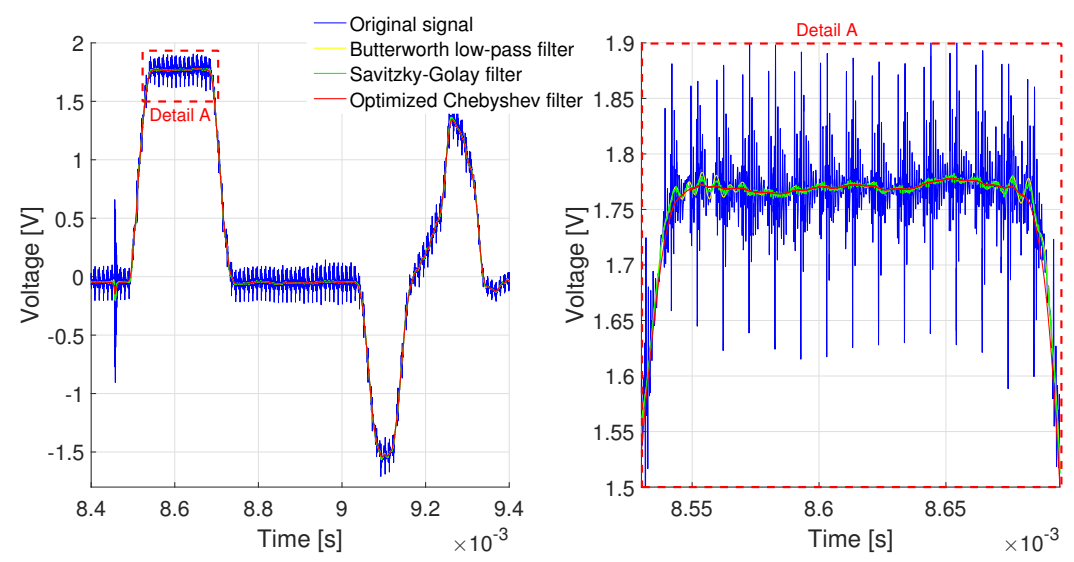

Figure 8. Comparison between different filters for incident signal: $l_{s}=500 \mathrm{~mm}$ (long striker), $v_{s}=45.36 \mathrm{~ms}^{-1}(\mathrm{high}$ striker velocity)
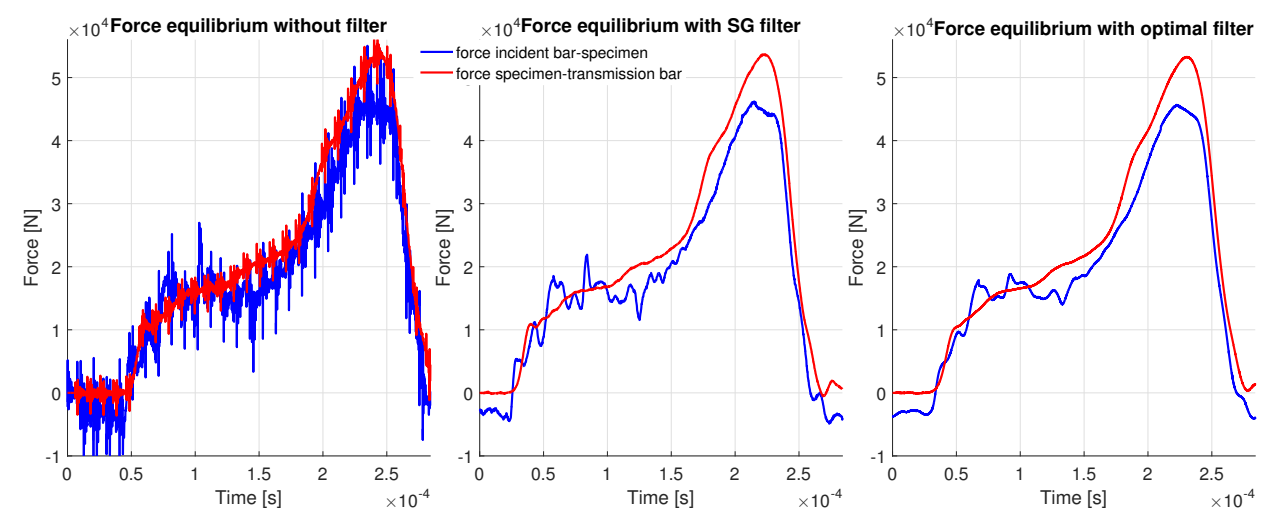

Figure 9. Comparison of force equilibrium obtained without signal filtering and with two different filters: $l_{s}=500 \mathrm{~mm}$ (long striker), $v_{s}=45.36 \mathrm{~ms}^{-1}$ (high striker velocity)

In Fig. 10 an effect of optimized filter application on strain equilibrium is shown. It was shown that optimized filter effect on experimental results was essential. Smoothing effect of both Butterworth and SG filter is not sufficient for evaluation of experiment equilibrium. It was also shown, that optimized filter is universal for all SHPB experiment signals.

This filter was previously used mainly for its simple implementation and input parameter modification. However it is not possible to derive an optimal solution for wide range of SHPB experiments, because its input parameters can not be directly linked with spectral analysis of signal and thus can deform it or filter out important information from it (e.g. mechanical oscillations in the measurement bars).

\subsection{Chebyshev filter (Type 2)}

Combination of three inverse Chebyshev band stop signal processing filters was used for optimization of SHPB signals filtration process. This type of filter was chosen mainly because of its maximally flat response in pass band and equiripple in stop band. This property could be used to design two notch filters (filters with very narrow stop band range) to attenuate two most important noise frequencies present in all measured signals shown in previous chapter. There are other less important singular noise frequencies present in measured signals, as can be seen in Fig. 5 7. but adding another component to designed filter did not improve the overall outcome of filtration. Those two frequencies were identified to be $f_{1}=639 \mathrm{kHz}$ and $f_{2}=980 \mathrm{kHz}$. For both frequencies attenuation of $A_{s}=70 \mathrm{~dB}$, filter order was 2. The third part of designed filter was designed again as an inverse Chebyshev filter band stop in range from $2 \mathrm{MHz}$ to $4 \mathrm{MHz}$ with attenuation of $A_{s}=20 \mathrm{~dB}$ and same filter order. It is shown, that this optimized filter fits experimental data in peak values and also visually provides the best results compared to other tested filters (Fig. 8, detail A).

\section{Results}

Evaluation of experimental SHPB data was performed both with and without pre-processing of signals using optimized filter. For comparison low-pass Butterworth filter with $250 \mathrm{kHz}$ passband and attenuation of $80 \mathrm{~dB}$ (which effectively attenuates lower frequencies without deforming measured signal, but does not smooth signals in acceptable fashion) and Savitzky-Golay fil- 


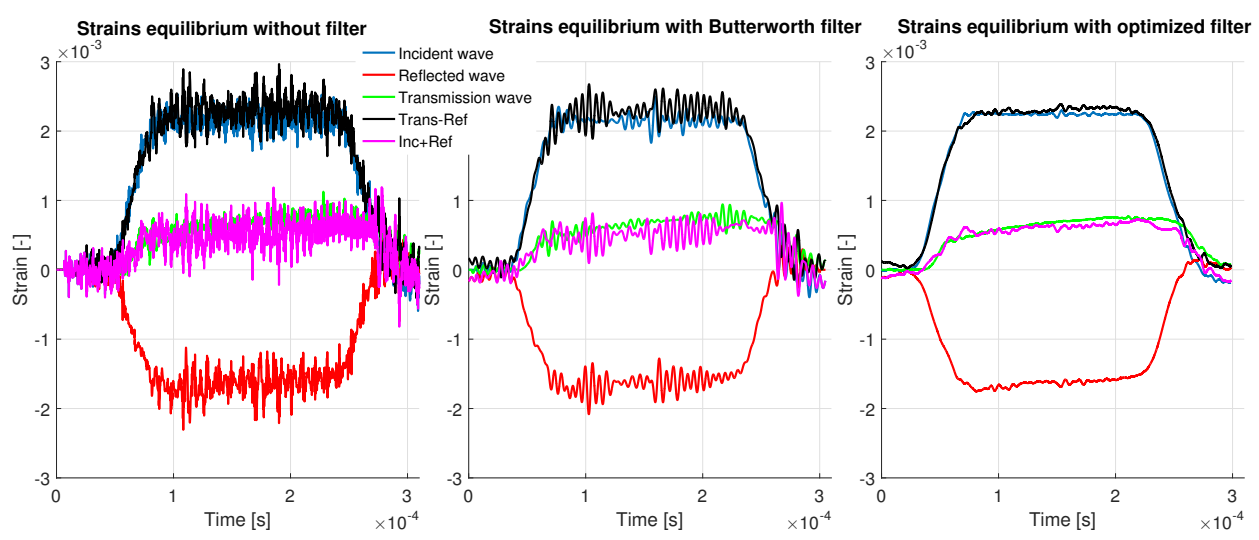

FiguRE 10. Comparison of strain equilibrium obtained without signal filtering and with two different filters: $l_{s}=500 \mathrm{~mm}$ (long striker), $v_{s}=24.05 \mathrm{~ms}^{-1}$ (high striker velocity)

ter with polynomial degree 2, frame width 99 (low polynomia degree was chosen over higher polynomial, see [6] and [8]). In Fig. 9 an effect of optimized filter application on force equilibrium as one of the main results of SHPB test evaluation is shown.

\section{CONClusion}

Analysis of measured SHPB signals was performed. It was shown that noise properties of SHPB signals were independent on the length of used striker and its impact velocity. It was also shown that noise properties are the same for both incident and transmission bar. Random noise present in the measured signals was therefore identified using spectral analysis. This noise (consisting of several singular noise frequencies) was identified to be only of the electric character. To filter the noise, optimal filter which minimized deformation of signal spectrum was designed based on inverse Chebyshev filtering procedure. Designed filter performance was compared with two different filtering methods which were both previously being used for SHPB signals pre-processing. Results of several experiments with and without applied filter were shown. To conclude, the optimized filtration method for pre-processing of SHPB data was introduced. The method was suitable for automated data filtering of signal from aluminum bars low impedance materials SLS manufactured auxetic lattice.

\section{ACKNOWLEDGEMENTS}

The research was supported by the Czech Science Foundation (project no. 15-15480S) and by Grant Agency of the CTU in Prague (grants no. SGS18/153/OHK2/2T/16 and SGS17/148/OHK2/2T/16). All the financial support is gratefully acknowledged.

\section{REFERENCES}

[1] A. Jung, S. Diebels. Microstructural characterisation and experimental determination of a multiaxial yield surface for open-cell aluminium foams. Materials and Design 131:252-264, 2017. DOI:10.1016/j.matdes.2017.06.017

[2] T. Fila, P. Zlamal, O. Jirousek, et al. Impact testing of polymer-filled auxetics using split hopkinson pressure bar. Advanced Engineering Materials 19(10), 2017. DOI:10.1002/adem.201700076.

[3] G. Sunny, F. Yuan, V. Prakash, J. Lewandowski. Design of inserts for split-hopkinson pressure bar testing of low strain-to-failure materials. Experimental Mechanics 49(4):479-490, 2009. DOI:10.1007/s11340-008-9145-1.

[4] P. Koudelka, O. Jirousek, T. Fila, T. Doktor. Compressive properties of auxetic structures produced with direct 3d printing. Materiali in Tehnologije 50(3):311-317, 2016. DOI:10.17222/mit.2014.204

[5] A.-X. Zhao, X.-J. Tang, Z.-H. Zhang, J.-H. Liu. The parameters optimization selection of savitzky-golay filter and its application in smoothing pretreatment for FTIR spectra. pp. 516-521. 2014. DOI:10.1109/ICIEA.2014.6931218.

[6] B. Zimmermann, A. Kohler. Optimizing savitzky-golay parameters for improving spectral resolution and quantification in infrared spectroscopy. Applied Spectroscopy 67(8):892-902, 2013. DOI:10.1366/12-06723

[7] M. Jakubowska, W. Kubiak. Adaptive-degree polynomial filter for voltammetric signals. Analytica Chimica Acta 512(2):241-250, 2004. DOI:10.1016/j.aca.2004.03.007

[8] P. Barak. Smoothing and differentiation by an adaptive-degree polynomial filter. Analytical Chemistry 67(17):2758-2762, 1995. DOI:10.1021/ac00113a006 